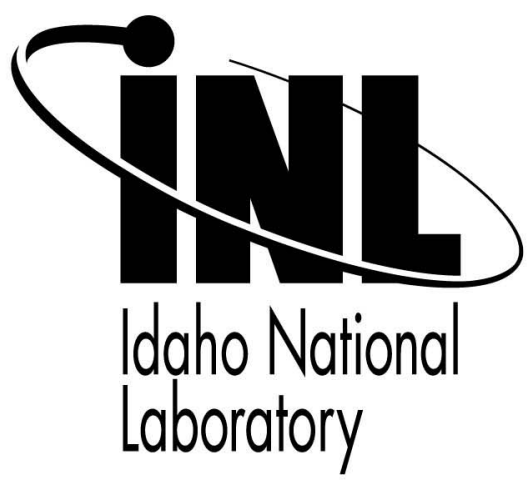

Joint 8th Annual Conference on Human Factors and Power Plants and the 13th Annual Workshop on Human Performance / Root Cause / Trending / Operating Experience / and Self Assessment (HPRCT)

\author{
April M. Whaley \\ Ronald L. Boring \\ Harold S. Blackman \\ Patrick H. McCabe \\ Bruce P. Hallbert
}

\section{August 2007}

This is a preprint of a paper intended for publication in a journal or proceedings. Since changes may be made before publication, this preprint should not be cited or reproduced without permission of the author. This document was prepared as an account of work sponsored by an agency of the United States Government. Neither the United States Government nor any agency thereof, or any of their employees, makes any warranty, expressed or implied, or assumes any legal liability or responsibility for any third party's use, or the results of such use, of any information, apparatus, product or process disclosed in this report, or represents that its use by such third party would not infringe privately owned rights. The views expressed in this paper are not necessarily those of the United States Government or the sponsoring agency. 


\title{
Lessons Learned from Dependency Usage in HERA: Implications for THERP-Related HRA Methods
}

\author{
April M. Whaley, Ronald L. Boring, Harold S. Blackman, Patrick H. McCabe, Bruce P. Hallbert \\ ${ }^{1}$ Idaho National Laboratory, Idaho Falls, Idaho, USA \\ \{april.whaley,ronald.boring,harold.blackman,patrick.mccabe,bruce.hallbert\}@inl.gov
}

\begin{abstract}
Dependency occurs when the probability of success or failure on one action changes the probability of success or failure on a subsequent action. Dependency may serve as a modifier on the human error probabilities (HEPs) for successive actions in human reliability analysis (HRA) models. Discretion should be employed when determining whether or not a dependency calculation is warranted: dependency should not be assigned without strongly grounded reasons. Human reliability analysts may sometimes assign dependency in cases where it is unwarranted. This inappropriate assignment is attributed to a lack of clear guidance to encompass the range of scenarios human reliability analysts are addressing. Inappropriate assignment of dependency produces inappropriately elevated HEP values. Lessons learned about dependency usage in the Human Event Repository and Analysis (HERA) system may provide clarification and guidance for analysts using THERP-based dependency models. This paper presents the HERA approach to dependency assessment and discusses considerations for dependency usage in HRA, including the cognitive basis for dependency, direction for determining when dependency should be assessed, considerations for determining the dependency level, temporal issues to consider when assessing dependency, (e.g., considering task sequence versus overall event sequence, and dependency over long periods of time), and diagnosis and action influences on dependency.
\end{abstract}

\section{INTRODUCTION}

The Human Event Repository and Analysis (HERA) system [1] is a tool developed to analyze and classify human performance information from nuclear power plant operating experience (e.g., event reports). The HERA system was specifically designed to be of a content and form useful to a variety of human reliability analysis (HRA) methods and the general discipline of human factors. As such, it consists of an analysis method that identifies information of interest to HRA, such as unsafe human actions, successful human actions including recoveries, performance shaping factors that contribute to human performance, and dependency between unsafe human actions.

\section{A. Dependency Assessment in HRA}

Dependency occurs when the probability of success or failure on one action changes the probability of success or failure on a subsequent action. The process of assessing dependency and incorporating dependency into the final human error probability (HEP) estimate varies by method. There is a wide variety of
HRA methods available for use today; some explicitly model dependency, some assess dependency as part of the larger context surrounding human performance, and others do not consider dependency at all. The dependency model used in the Technique for Human Error Rate Prediction (THERP) [2] HRA method is widely used on its own and serves as the foundation for dependency modeling in other widely used methods such as SPAR-H [3] and ASEP [4].

Chapter 10 of THERP [2] provides an extensive discussion of dependency, including defining dependency levels ranging from zero dependence to complete dependence. The THERP process breaks down tasks into subtasks through task analysis, and provides explicit instructions for assessing dependencies among subtasks. It does not, however, provide guidance for considering dependency between different tasks. The subtask dependency model is often used to address dependency between tasks in THERP, which may not always be appropriate.

NUREG-1792, Good Practices for Implementing Human Reliability Analysis (HRA) [5], emphasizes examining human performance throughout the entire accident sequence for commonalities, similarities, and links among the actions, such as when involving the same crew members, occurring closely in time, sharing a common mindset, etc. In other words, the Good Practices document advocates considering the context of the actions in question when assessing dependency. The THERP dependency model only accounts for a small set of factors when calculating dependency, and therefore would not fully conform to the guidance established for dependency assessment in the Good Practices document. Because THERP does not fully consider context, using the THERP model of dependency may result in unrealistic HEP values.

Inappropriate dependency assignment may be attributed to a lack of clear guidance to encompass the range of scenarios human reliability analysts are addressing. Additionally, guidance regarding issues such as how to determine when dependency should be assessed, how to determine the factors or mechanisms that lead to dependency and how to consider those factors when assigning the dependency level, and how to properly consider temporal issues when assessing dependency is insufficient or absent [6]. Lessons learned about dependency usage in developing the HERA system and analyzing events within the HERA system may provide clarification and guidance about these issues. 


\section{HERA GUIDANCE ON DEPENDENCY ISSUES IN HRA}

\section{A. The HERA Approach to Dependency}

The HERA approach to dependency is based in part on the Good Practices in HRA document [5], but it differs slightly from the standard processes used in individual HRA methods, as it was designed to be informative to HRA in general, regardless of the specific method being employed. As HERA was being developed, it became clear that the approach to dependency offered by the many HRA methods was not appropriate for the data used in HERA. The information available in event reports does not lend itself easily to dependency calculation. In many cases, event reports offer a high-level overview of risk-significant activities that may omit risk-insignificant steps or contributors to the event sequence, therefore offering an account that may obscure any dependencies between human actions. Therefore, the HERA dependency assessment process was modified from traditional HRA dependency assessment to better suit the data being analyzed.

The HERA analysis process breaks down an event report into a timeline of subevents, individual human and equipment operations, and actions that contribute to an overall event. A human action that potentially decreases the safety of the event, such as an error, is classified as an "XHE" in HERA. The definition of dependency used in HERA is, "dependency exists between two subevents when an error on one subevent increases the probability that an error will occur on a subsequent subevent" [7].

HERA recognizes that dependency can exist between successful human actions and errors. However, because most HRA methods do not currently model the relationship between human errors and successful human actions, dependency is only completed for potentially unsafe human subevents, or XHEs. The approach to dependency assessment adopted in HERA does not attempt to quantify the level of dependency, nor does it limit the parameters that need apply. Instead, HERA analysts determine if dependency exists between two subevents and then identify the source of dependency (i.e., explain what dependence mechanisms were involved).

\section{B. Determining Whether Dependency Should Be Assessed}

The first step an analyst should take when considering dependency between human actions is to determine whether a dependency calculation is warranted. In practice, however, it is common for analysts performing an HRA to automatically perform a calculation of dependency level without first questioning whether dependency is actually present between the actions under consideration. The HERA analysis process instructs analysts to first consider whether there is any reason for dependency to occur between human actions.

The HERA authors believe that dependency between human actions is largely based in human cognition: dependency between human actions arises from the knowledge or lack of knowledge of the person involved in the second task regarding the occurrence and/or effect of the previous task. If an operator has no knowledge of a prior task, then it is not possible for that task to affect performance of a subsequent task. ${ }^{1}$ More clearly stated, dependency arises from mindset. Mental models are updated by experience, so prior actions and errors can act as current cues and create expectancies and predispositions to behave in a certain manner. In other words, previous actions or experiences create a mindset that directs decision making [3].

The HERA dependency assessment process considers the multitude of situational, contextual, organizational, cognitive, and personal factors that can affect task dependency. HERA instructs analysts to ask the following questions to help them make an appropriate decision as to whether dependency exists between two human actions:

- Do the actions under consideration involve similar tasks?

- Are they part of a related sequence of actions?

- Do the personnel involved in each task share knowledge or a mindset that guides their actions?

- Is there any reason to expect that the first action influences the second?

If several subevents are all influenced by the same performance shaping factor (PSF), such as poor work processes, for example, it does not necessarily mean there is a dependency between them. If the PSF increases the likelihood of subsequent errors, then dependency exists. In other words, HERA asks analysts to fully consider the context of the actions under analysis and determine whether the situation is producing dependency between human actions.

In the case where multiple errors are made due to the same PSF but are otherwise unrelated to each other, HERA considers this to be a case of shared PSFs, not dependency, and identifies this information in the PSF assignment sections, not in the dependency section. HRA analysts also should consider these issues in the context of the method they are using before making a dependency calculation. An obvious example is that dependency is unlikely to occur between human actions that are a part of separate functions on an event tree, because the actions are unrelated to each other. Generally speaking, if there is no reason to predict that an error on one task or action makes a subsequent error more likely, then dependency does not exist between the two actions.

\section{Determining Dependency Level: Dependency Factors}

The traditional THERP [2] approach to dependency assessment uses several parameters to determine the level of dependency between subevents, including same or different crew, time, location, and cues. With these parameters came a scale that rated dependency from zero (no dependency) to a value representing complete dependency (see Table I). Following these criteria too rigidly, however, may lead to assignments of dependency levels that do not make sense given the situation under analysis, or are otherwise inappropriate. For example, not considering the possibility of zero dependency, even when all dependency factors do not apply, could

\footnotetext{
The exception to this informal rule comes in cases when the operator should have been cognizant of a required action but failed to perform it. Such an error of omission may propagate itself, as the operator may continue not to realize
} the error, subsequently hindering successful recovery actions. 
erroneously result in the assignment of low dependency using the guidance in Table I.

TABLE I. DEPENDENCY ASSIGNMENT FROM SPAR-H BASED ON THERP.

\begin{tabular}{|c|c|c|c|c|c|}
\hline $\begin{array}{c}\text { Dependency } \\
\text { Level }\end{array}$ & $\begin{array}{l}\text { Same } \\
\text { Crew }\end{array}$ & $\begin{array}{l}\text { Close in } \\
\text { Time }\end{array}$ & $\begin{array}{c}\text { Same } \\
\text { Location }\end{array}$ & $\begin{array}{c}\text { No } \\
\text { Additional } \\
\text { Cues }\end{array}$ & $\begin{array}{l}\text { Dependency } \\
\text { Modification }\end{array}$ \\
\hline \multirow[t]{2}{*}{ Complete } & $\checkmark$ & $\checkmark$ & $\checkmark$ & $\checkmark$ & \multirow[t]{2}{*}{$\mathrm{HEP}=1$} \\
\hline & $\checkmark$ & $\checkmark$ & $\checkmark$ & & \\
\hline \multirow{3}{*}{ High } & $\checkmark$ & $\checkmark$ & & $\checkmark$ & \multirow{3}{*}{$\frac{1+\mathrm{HEP}}{2}$} \\
\hline & $\checkmark$ & $\checkmark$ & & & \\
\hline & $\checkmark$ & & $\checkmark$ & $\checkmark$ & \\
\hline \multirow[t]{6}{*}{ Moderate } & $\checkmark$ & & $\checkmark$ & & \multirow{6}{*}{$\frac{1+6 \times \mathrm{HEP}}{7}$} \\
\hline & $\checkmark$ & & & $\checkmark$ & \\
\hline & & $\checkmark$ & $\checkmark$ & $\checkmark$ & \\
\hline & & $\checkmark$ & $\checkmark$ & & \\
\hline & & $\checkmark$ & & $\checkmark$ & \\
\hline & & $\checkmark$ & & & \\
\hline \multirow[t]{4}{*}{ Low } & $\checkmark$ & & & & \multirow{4}{*}{$\frac{1+19 \times \mathrm{HEP}}{20}$} \\
\hline & & & $\checkmark$ & $\checkmark$ & \\
\hline & & & $\checkmark$ & & \\
\hline & & & & $\checkmark$ & \\
\hline Zero & & & & & HEP \\
\hline
\end{tabular}

HERA does not quantify the level of dependency, nor does it limit the parameters that can produce dependency. Instead, HERA analysts consider the context surrounding the subevents in question, determine whether dependency exists between two human actions and then explain the factors or mechanisms that led to the dependency (i.e., describe the context). HERA recognizes the THERP factors listed above (crew, time, location, and cues) as mechanisms for dependency, but these are only a subset of a much larger set of potential dependency mechanisms or contextual factors that can lead to dependency as well. A list of some possible contextual dependency factors that are included in HERA is presented in Table II below. Note that these mechanisms overlap one another and should not be considered orthogonal. Also note that this list is not exhaustive; HERA analysts are instructed to carefully consider any factor that reasonably triggers an increased likelihood of a negative outcome across subevents as a candidate dependency mechanism. It is important for HRA analysts to be aware that the factors presented in HRA methods as criteria for determining the dependency level are not absolute rules. Two actions can occur closely in time, be in the same location, and involve the same personnel, but be independent because the actions are part of unrelated tasks or functions. Similarly, moderate dependency can exist between two actions despite involving different personnel across a long period of time.

Currently the HERA system is designed primarily for retrospective analyses. Analysts performing a prospective HRA have to predict the most likely contributing factors and whether dependency is at play. Often, HRA analysts will set a high level of dependency as a screening value to determine the strongest possible or worst case effect of dependency on the total HEP. Our advice to HRA analysts performing prospective analyses is similar to that for retrospective analyses: analysts should carefully consider the situation under review, the tasks involved, the possible mechanisms for dependency, and the
THERP definitions of the dependency levels, and then decide whether a dependency calculation is warranted and which level of dependency is most appropriate for the situation.

\section{Proper Consideration of Temporal Sequence}

HERA recognizes that dependency can occur between human actions that are not contiguous in time. It is possible for series of parallel activities to occur during an event. In such a case, the dependencies should reflect the proper track of occurrence, even when actions from different tracks co-mingle chronologically. HERA analysts consider dependency among subevents within tasks, rather than between subevents that are adjacent in the chronological order but are a part of separate tasks. For example, in Fig. 1 below, XHE 1 and XHE 3 are part of the same task sequence, and it is possible for XHE 3 to be dependent on XHE 1. XHE 2, however, represents a parallel activity that is unrelated to the task sequence including XHE 1 and XHE 3. Dependency occurs only between XHE 1 and XHE 3 , even though XHE 2 occurs between them chronologically.

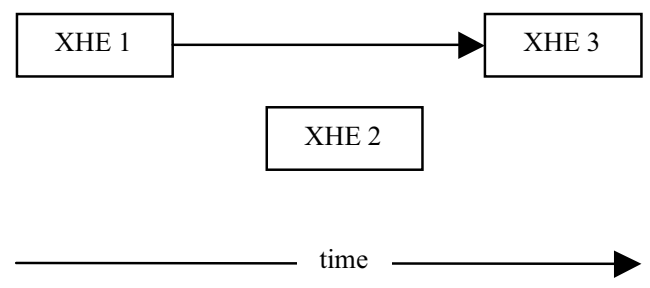

Figure 1. Task sequence vs. chronological sequence.

In order to properly consider possible dependencies between all XHEs, HERA analysts use the dependency matrix when determining whether dependency exists between two XHEs (see Fig. 2). Each XHE in the event under analysis is listed across the top row and down the left column, in chronological order. This allows HERA analysts to indicate whether dependency exists between the first XHE (listed in the column) and the second, the third, and so on (listed in the top row), by checking the box that intersects the two subevents. Using the list of possible dependency mechanisms (see Table II) as a guide, the HERA analyst moves to the second row and indicates any dependency between the second XHE and the third, the fourth, and so on, continuing down the rows until all subevents are accounted for.

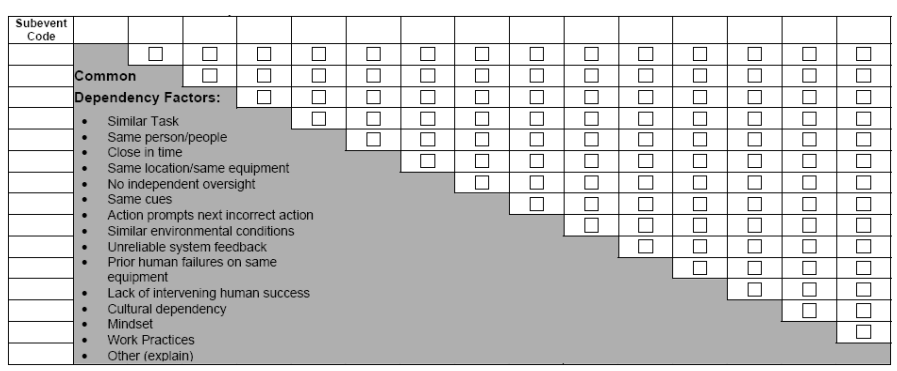

Figure 2. The HERA Dependency Matrix. 
TABLE II. SELECTED HERA DEPENDENCE MECHANISMS

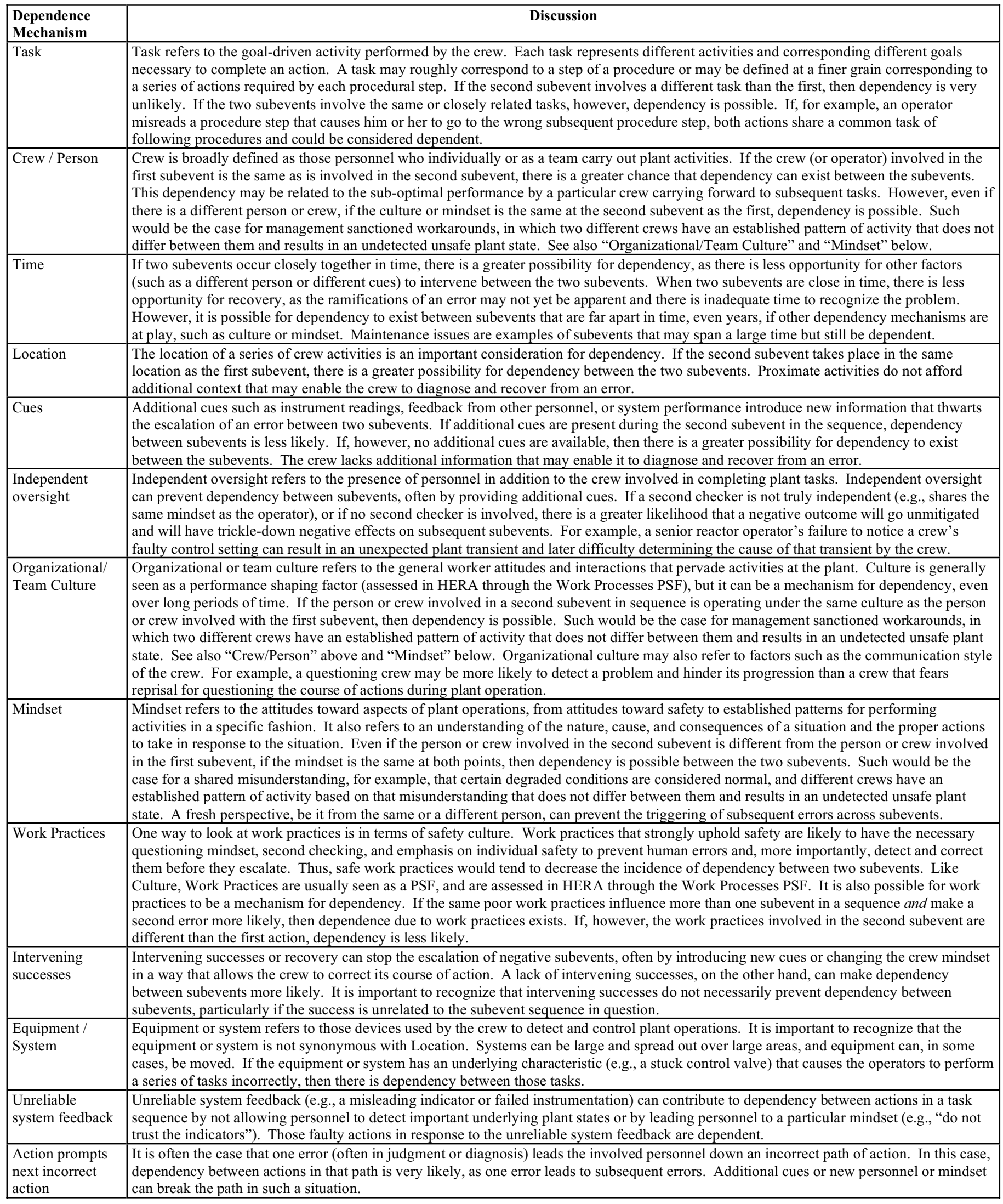


Human reliability analysts often work from an event tree, in which case they are considering human actions within a task sequence. However, analysts typically only consider dependency between successive actions in a sequence (e.g., between XHE 1 and XHE 2, between XHE 2 and XHE 3, etc.). HERA allows consideration of dependency between XHE 1 and XHE 4, something that it not routinely performed in HRA currently (see Fig. 3). Whether working from an event tree or not, HRA analysts should be aware of the need to work within task sequences and to identify possible dependencies between human actions that they would not normally consider.

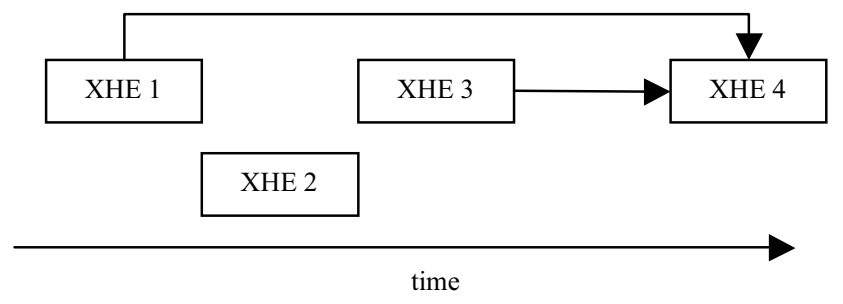

Figure 3. Dependency throughout an action sequence

Dependencies over very long timeframes are generally not addressed by current HRA methods. However, multiple HERA analyses have identified dependencies between subevents that occurred years apart. Such cases have often been due to instances of a shared organizational culture or mindset that led to increased unsafe actions across time. Latent errors-those that do not have an immediate impact but may affect system safety at a later time - almost invariably feature some degree of dependency over a long timeframe. The HERA approach to dependency allows analysts to identify and describe such cases of dependency over long periods of time. HRA analysts should remain aware of the possibility of dependency over long timeframes, and include such calculations in their analyses as appropriate.

\section{E. Implications of Diagnosis and Action for Dependency Assessment}

Some HRA methods such as SPAR-H [3] weight PSFs differently depending on whether the subevent under analysis involves diagnosis (a cognitive task such as decision making) or action (a behavioral response such as activating a switch), but there is little guidance available on how to properly consider diagnosis and action tasks when assessing dependency. Further research is needed to determine exactly how diagnosis and action tasks influence dependency. Insights gained from the HERA system may provide some clarification and serve to generate future exploration of this issue. The following argument is presented as a topic for further discussion.

HERA describes these four steps of human information processing or decision making [7]:

- Detection or recognition of a condition or change in situation (e.g., a problem or alarm)

- Interpretation of the condition or change in situation

- Planning a response to the situation

- $\quad$ Executing the response (action)
Diagnosis consists of the first three steps. Errors can occur at any of these steps and at more than one step in the sequence. In HERA, it is possible to see an XHE represent all of these steps, or for several XHEs to occur within one sequence.

One of the dependency mechanisms listed in Table II above is "Action prompts next incorrect action." This refers to the case where an error early on in the human information processing steps leads personnel down an incorrect path of actions. If an error is made in interpretation of the situation, subsequent planning and actions are more likely to be inappropriate; they are more likely to be dependent upon that error in interpretation. On the other hand, if the first error is an error in action implementation, any subsequent errors or unsafe actions are more likely to be unrelated to the first error. Recall the discussion above about dependency arising from mindset. The diagnosis steps of this process establish the mindset, and then the action follows. If the diagnosis is correct but an error occurs in response implementation, operators are likely to recognize the error and take actions to correct it. If however, the error is in diagnosis, actions taken as a result of the incorrect mindset are less likely to be recognized as inappropriate.

The HERA authors propose that when two actions under consideration for dependency are a part of the same detectioninterpretation-planning-response sequence, then dependency is likely. If the two actions are part of separate, unrelated cognitive sequences, dependency is not likely. In many ways, this is similar or parallel to the THERP approach of considering dependency between subtasks within a task. Within the steps of the human information processing, or within a task, dependency is more likely than between separate cognitive sequences or tasks, respectively. The information processing process is something that is repeated iteratively. It is possible for two actions to be part of separate cognitive sequences that are related in a larger series of human actions. In such a case, dependency between the two actions is possible.

\section{F. Dependency and HEP Calculations}

Some HRA methods such as SPAR-H [3] provide explicit instructions on how to calculate dependency levels and include dependency in the HEP calculation. Other HRA methods leave assessing a dependency level and incorporating dependency calculation into the HEP estimate to analyst discretion [6]. HERA does not assign dependency levels, as discussed above, nor does it generate HEP values. For HRA methods that assign a dependency level, the HERA authors recommend that HRA analysts review the descriptions of the dependency levels provided in THERP [2], consider the factors and processes included in the HRA method being employed, consider the additional dependency mechanisms discussed in HERA, and consider the full context of the actions in question when assigning a dependency level. Our advice is similar for determining how to incorporate dependency into the overall HEP value: HRA analysts should give full consideration to the context of the actions under analysis within the process of the method being employed. Such a careful approach will enable analysts to avoid overestimating or underestimating the dependency level between two human actions. 


\section{DISCUSSION}

THERP-based dependency models lack consideration of some key contextual factors that are importance in today's HRA modeling. Yet they are still widely used. Issues relating to insufficient guidance regarding dependency assessment may result in inconsistent and/or inappropriate HEP values. Insights gained from the HERA system and approach to dependency provide additional clarification and guidance for dependency assessment in cases where existing information may not apply, and by bringing the consideration of context to THERP-based dependency models, may therefore improve the HRA dependency assessment process and resultant HEP values. HERA provides discussion for determining when dependency should be assessed, information about a variety of factors that lead to dependency between human actions to aid analysts in determining the proper dependency level, discussion about temporal issues that affect dependency calculations, and guidance for properly considering diagnosis and action when assessing dependency. Armed with guidance from the HRA Good Practices [5], and guidance from HERA [7], HRA analysts will be better prepared to appropriately and accurately characterize and include dependency in their HEP calculations.

\section{DISCLAIMER}

This article was prepared as an account of work sponsored by an agency of the US Government. The opinions expressed in this paper are those of the authors and not of an agency of the US Government. Neither the US Government nor any agency thereof, nor any employee, makes any warranty, expressed or implied, or assumes any legal liability or responsibility for any third party's use, or the results of such use, of any information, apparatus, product, or process disclosed in this publication, or represents that its use by such third party would not infringe privately owned rights. This work was supported by the US Nuclear Regulatory Commission under US Department of Energy Idaho Operations Contract DE-AC07-05ID14517.

\section{REFERENCES}

[1] B. Hallbert, R. Boring, D. Gertman, D. Dudenhoeffer, A. Whaley, J. Marble, J. Joe, and E. Lois, Human Event Repository and Analysis (HERA) System, Overview, NUREG/CR-6903, Vol. 1, Washington, DC: US Nuclear Regulatory Commission, July 2006.

[2] A. D. Swain, and H. E. Guttman, Handbook of Human Reliability Analysis with Emphasis on Nuclear Power Plant Applications, NUREG/CR-1278, Washington, DC: US Nuclear Regulatory Commission, August 1983.

[3] D. Gertman, H. Blackman, J. Marble, J. Beyers, and C. Smith, The SPAR-H Human Reliability Analysis Method, NUREG/CR-6883, Washington, DC: US Nuclear Regulatory Commission, August 2005.

[4] A. D. Swain, Accident Sequence Evaluation Program (ASEP) Program, NUREG/CR-4772, Washington, DC: US Nuclear Regulatory Commission, February 1987.

[5] A. Kolaczkowski, J. Forester, E. Lois, and S. Cooper, Good Practices for Implementing Human Reliability Analysis (HRA), NUREG-1792, Washington, DC: US Nuclear Regulatory Commission, April 2005.

[6] J. Forester, A. Kolaczkowski, and E. Lois, Evaluation of Human Reliability Analysis Methods Against Good Practices, Draft Report for Public Comment, NUREG-1842, Washington, DC: US Nuclear Regulatory Commission, March 2006.

[7] B. Hallbert, A. Whaley, R. Boring, P. McCabe, and E. Lois, Human Event Repository and Analysis (HERA): The HERA Coding Manual and Quality Assurance, NUREG/CR-6903, Vol. 2, Washington, DC: US Nuclear Regulatory Commission, in press. 